\title{
ANGLE-RESOLVED UV PHOTOEMISSION FROM SINGLE CRYSTALS OF AgPd ALLOYS
}

\author{
L. ILVER AND P.O. NiLsson \\ Department of Physics, Chalmers University of Technology \\ 41296 Gothenburg, Sweden \\ Dedicated to Professor Dr. Julian Auleytner \\ on the occasion of his 70th birthday
}

(Received September 16, 1992)

The angular dependence of photoemission energy distributions were measured from single crystal, substitutional disordered alloys $\operatorname{Ag}_{x} \operatorname{Pd}_{1-x}$ with $x=0.97$ and $x=0.90$. The random hybridization between the $\mathrm{Ag} 5 s p$ band and the $\mathrm{Pd} 4 d$ state is discussed in terms of complex energies.

PACS numbers: 79.60.Gs, 71.25.Pi

\section{Introduction}

The electronic structure of elemental solids, compounds and ordered alloys can today be calculated to a high accuracy by first principles theories, see for instance [1]. The translation invariance in such systems simplifies the description considerably. In disordered systems, e.g. substitutionally disordered alloys, this simplification is removed and the electron states cannot be described by conventional Bloch waves. The description of random alloys has been developed into quite sophisticated theories during the last 20 years, since the pioneering paper by Soven [2]. A central concept is the coherent potential approximation, CPA. This is a mean-field theory and one usually applies a single-site approximation. Advanced numerical methods have been developed to solve the CPA problem in a KKR formalism $[3,4]$. Comparisons have been made with experimental density-of-states related functions obtained from e.g. photoelectron spectroscopy. However, the theory provides much more information than this. In particular, Bloch spectral densities $A(k, E)$ are available for a number of alloy systems. Only in a few cases however there have been attempts to correlate these data with experimental results. We believe that it is very important to make detailed comparisons between theory and experiment to test the present CPA theories.

In the present work we focus our attention on a simple, but fundamental situation, namely a transition metal impurity in a free-electron-like host. The 
impurity limit of this problem was formulated [5] and tested [6] a long time ago in terms of a "virtual bound state" model. CPA calculations provide however a much more detailed description of such a system, which to our knowledge has not yet been subject to detailed experimental tests. New data are presented here and analysed using a simple model calculation. The data should however also be valuable for comparisons with full CPA calculations.

\section{Experimental}

The elastic photoelectrons originate from the outermost atomic layers of the sample. Because of this, photoemission experiments require a high-quality sample surface. Most alloy studies in literature have been performed on surfaces cleaned by ion bombardment and heating. With such techniques it can be difficult to obtain well-defined surface compositions. This problem is to a large extent eliminated in our studies where the crystals were fabricated in situ by simultaneous evaporation of $\mathrm{Pd}$ and $\mathrm{Ag}$ onto a $\mathrm{Ag}(111)$ substrate at room temperature. The alloy films were found to grow epitaxially. Calibration of the sources was done by measuring thicknesses of pure $\mathrm{Ag}$ and $\mathrm{Pd}$ films by means of a quartz oscillator. The evaporation rates were of the order of one atomic layer per minute. The final concentrations $x=0.97$ and $x=0.90$ were determined by examining the signal from the Pd impurity in the spectra.

A dedicated equipment for photoemission experiments was constructed. The light from a hydrogen discharge lamp was passed through a VUV monochromator and focused by an ellipsoidal mirror onto the sample. The light spot was approximately $2 \mathrm{~mm}$ wide. Energy distribution curves (EDC's) were recorded for sets of emission angles at a photon energy of $h \nu=10.2 \mathrm{eV}$, using a cylindrical sector analyser.

\section{Results}

In Fig. $1 \mathrm{EDC}$ 's for $\mathrm{Ag}_{0.9} \mathrm{Pd}_{0.1}$ are shown. The electron detection angle was varied in the range $0-35^{\circ}$ relative to the surface normal. For high angles a peak labelled $d$ is observed around $2.0 \mathrm{eV}$ below the Fermi level. Its energy does not vary with the angle. This "virtual bound state", associated with the $4 d$ level of Pd, has been reported earlier in a number of studies, see e.g. Ref. [6]. Two dispersing peaks $s p 1$ and $s p 2$ are also detected. These are associated with the free-electron-like $s p$ band of the Ag host. The peak $s p 2$ is well defined for low angles but becomes very weak for high angles. It is not possible to give an exact limit when it disappears. The peak $s p 1$ is most easily detected at high angles.

The peak positions are plotted in Fig. 2. There are also shown the results for pure Ag. The angular parameter was converted to the $k$-vector component parallel to the surface, $\boldsymbol{k}_{\|}$. We observe that the Pd impurities are strongly influencing the $E-k$ dispersion of the $s p$ band. For an alloy with $x=0.97$, not shown in the figure, the dispersion was almost unaffected. 


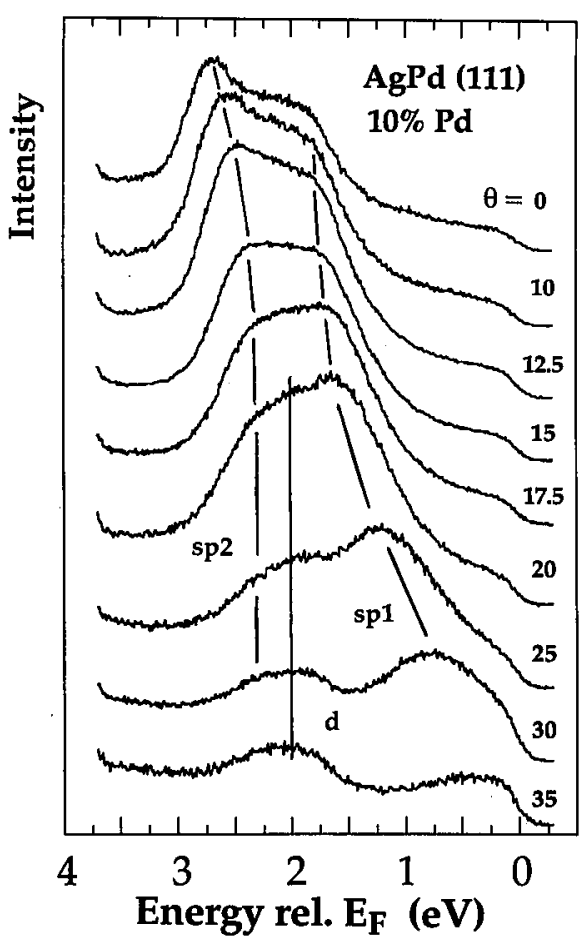

Fig. 1. Photoelectron energy distributions from $\mathrm{Ag}_{0.9} \mathrm{Pd}_{0.1}$ for emission angles $0-35^{\circ}$ towards $[11 \overline{2}]$ and photon energy $h \nu=10.2 \mathrm{eV}$.

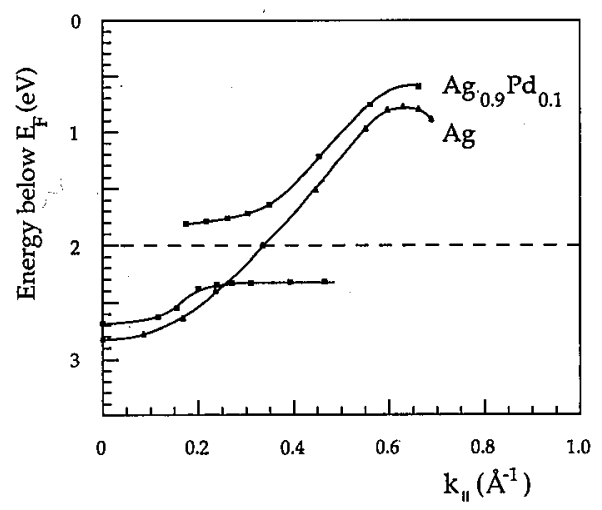

Fig. 2. Energies of the structures in Fig. 1 as a function of the $k$ vector parallel to the surface.

\section{Theory}

Multiple scattering theory is an adequate theoretical framework for the description of the electronic structure of solids. A central concept in this connection 
is the $t$-matrix $t\left(k, k^{\prime} ; E\right)$, which describes the scattering of an electron from a state $k$ to a state $k^{\prime}$. Assuming elastic scattering $|k|^{2}=\left|k^{\prime}\right|^{2}$ and a spherically symmetric potential, the $t$-matrix is reduced to a scalar quantity proportional to the scattering amplitude [3]:

$$
f_{l}(E)=\sqrt{E} t_{l}(E)=\frac{1}{2 \mathrm{i}}\left[\exp \left(\mathrm{i} 2 \delta_{l}\right)-1\right] .
$$

The index $l$ is the angular momentum quantum number and the energy dependent real function $\delta_{l}$ is the phase shift.

In a substitutionally disordered alloy the potential fluctuates randomly. In a simple picture we may use an average, periodic potential as an approximation for the actual potential. In a better approximation the Bloch functions associated with this average potential are scattered by a random potential, which is the difference between the true random potential and the averaged one. This means that a Bloch state of given wave vector $\boldsymbol{k}$ has a finite lifetime. Using the scattering amplitude we can construct a model where an electron at a given site is scattered according to $t_{A, l}$ with a probability $c$, the concentration of constituent $A$. Similarly the electron scatters according to $t_{B, l}$ with a probability $1-c$. This, so-called average $t$-matrix approximation (ATA), gives an effective scattering

$$
\bar{t}=c t_{A}+(1-c) t_{B}
$$

which has to be described by a complex phase shift, or equivalently with a real phase shift as

$$
f_{l}(E)=\frac{1}{2 \mathrm{i}}\left[\alpha_{l}(E) \exp \left(\mathrm{i} 2 \delta_{l}\right)-1\right],
$$

where the real function $\alpha_{l}(E)$ is less than unity. This formally inelastic scattering induces a complex self-energy, the imaginary part of which is a measure of the inverse lifetime of the Bloch state. Due to the dispersion of the Bloch state a broadening will also occur in the $k$ space. The effect can be summarized by the spectral density $A(k, E)$. For an ordered crystal $A(k, E)$ reduces to $\delta$-functions describing the ordinary band structure.

The model can be further refined by going to self-consistency, i.e. by requiring that the average scattering from a given site should be the same as that from a complex "coherent potential", which is the same at all sites. In other words, there is no net scattering from the given site. Formula (2) now becomes more complicated. This is the so-called coherent potential approximation, CPA. It is still a single-site, a verage potential theory, but effects of e.g. cluster scattering may be incorporated.

CPA calculations have ben presented in literature on $\mathrm{Ag}_{x} \mathrm{Pd}_{1-x}$ [7] and similar systems such as $\mathrm{Cu}_{x} \mathrm{Ni}_{1-x}$ [8]. With regard to the present problem Pindor et al. [7] are able to theoretically follow the $s p$ band through the $\mathrm{Pd}$ resonance, however strongly damped. This behaviour is different from an ordered crystal, where symmetry considerations forbid band crossing.

We have considered a very simple parameterized model which reproduces the main qualitative features of the CPA results. The $\mathrm{Ag} 5 s p$ band is represented by a free-electron parabola $E=\hbar^{2} k^{2} / 2 m$ and the Pd $4 d$ state is taken as one atomic level of energy $E=E_{d}$. The hybridization is represented by a constant $W$. Pd metal has five $d$ electrons (not considering spin) which at the centre of the 
Brillouin zone form one twofold $\left(e_{2}\right)$ and one threefold $\left(t_{2 \mathrm{~g}}\right)$ state. Only the latter hybridizes with the $s p$ band, and has to be considered in our model calculations. The disorder introduces, in principle, complex self-energies in all terms. As we are far from the $\mathrm{Ag} 4 d$ resonance the $s p$ band damping is neglected, being much weaker than the strongly damped Pd $4 d$ state. For the latter we introduce the complex energy $E=E_{d}+\mathrm{i} V_{d}$. A complex $W$ just rescales the problem, therefore we consider a real $W$. The eigenvalue problem thus becomes

$$
\left|\begin{array}{cc}
\hbar^{2} k^{2} / 2 m-E & W \\
W & E_{d}+\mathrm{i} V_{d}-E
\end{array}\right|=0,
$$

which is solved for complex energies $E=E_{\mathrm{r}}+\mathrm{i} E_{\mathrm{i}}$. Figure 3 shows the complex band structure for different choices of the damping $V_{d}$. We have arbitrarily chosen
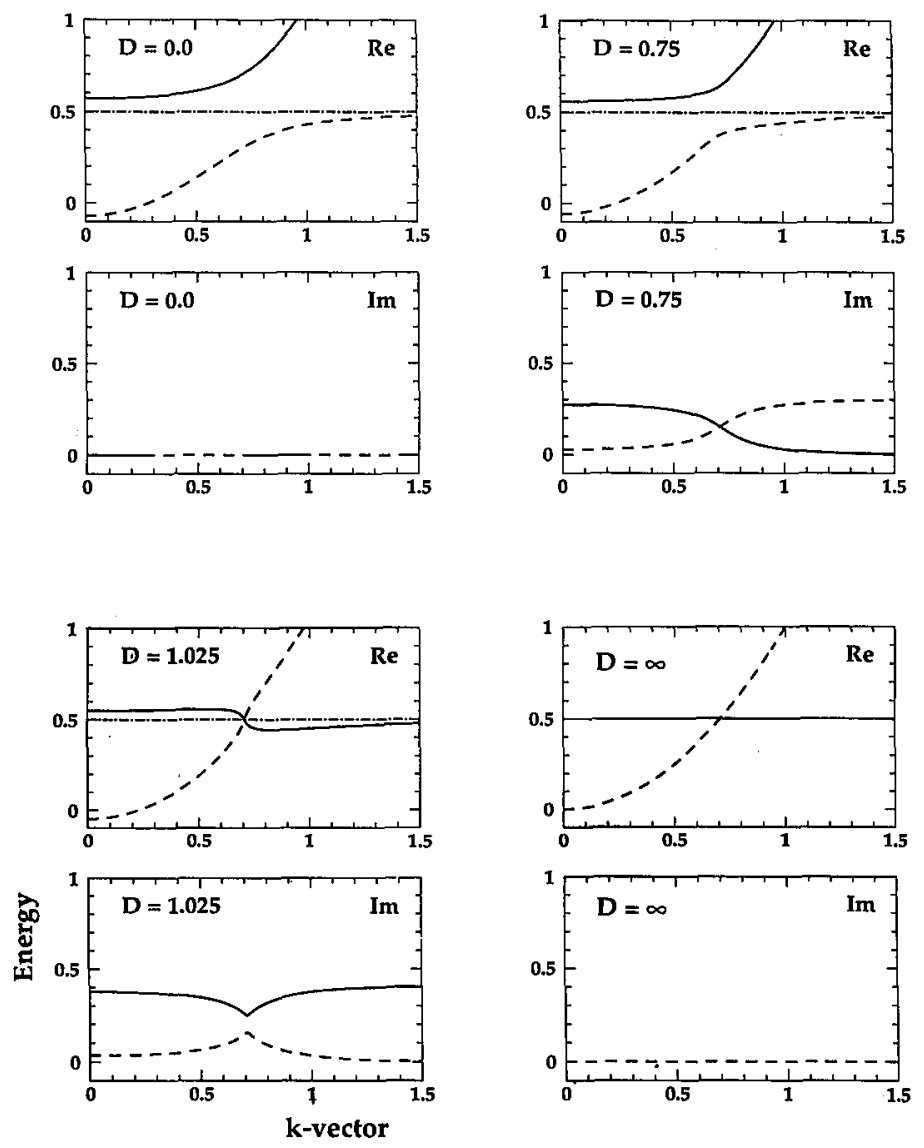

Fig. 3. Model calculations of the complex band structure of $\operatorname{Ag}_{x} \operatorname{Pd}_{1-x}$ for various dampings $D=V_{d} / 2 W$, where $V_{d}$ is the imaginary part of the Pd $t_{2 \mathrm{~g}} d$ energy and $W$ is the $s p-t_{2 g} d$ hybridization. Re and Im denote real and imasinary part of the energy. 


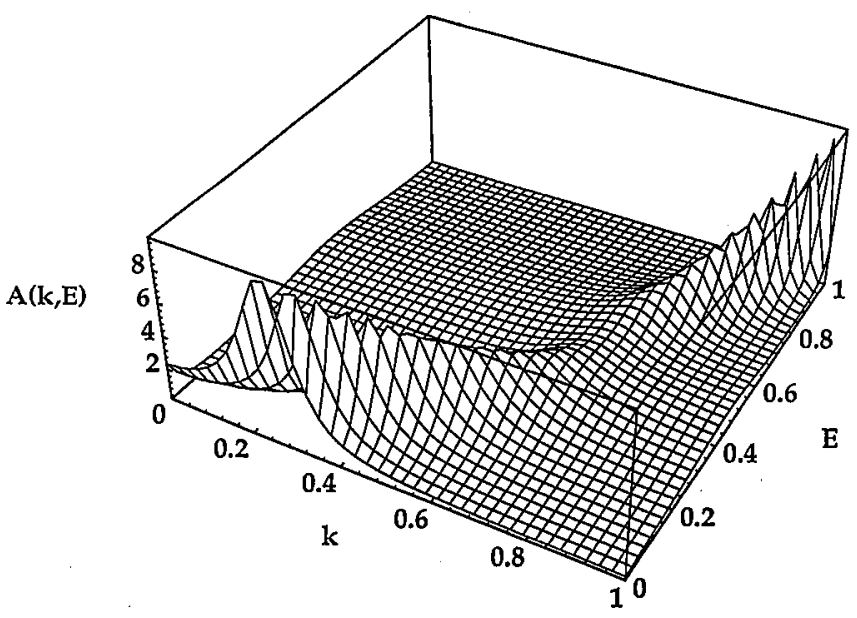

Fig. 4. The spectral function $A(k, E)$ for the case $D=0.75$ in Fig. 3 .

$\hbar^{2} / 2 m=1, W=0.2$ and $E_{d}=0.5$ and introduce the reduced damping $D=$ $V_{d} / 2 W$, where $2 W$ is the hybridization gap at $E_{d}=k^{2}(k=\sqrt{0.5}$ in our case). For low damping, $D<1$, the $s p$ and $d$ bands hybridize, but the gap diminishes with increasing $D$. For large damping, $D \geq 1$, the $\mathrm{Ag} s p$ band crosses the $\mathrm{Pd} d$ band. A distortion of the free-electron band is observed at the crossing. For very large damping, $D \rightarrow \infty$, we obtain the free-electron parabola $E=\hbar^{2} k^{2} / 2 m$, the $d$ state being totally damped out due to its short life time.

To get in contact with experimental data we calculated the spectral function $A(k, E)$, assuming that each band has a Lorentzian shape with a full width at half maximum (FWHM) being equal to the imaginary part of the self-energy. In Fig. 4 we show the result for $D=0.75$. The hybridization gap in $E_{\mathrm{r}}$ is not closed for this value of $D$ (see Fig. 3) but $A(k, E)$ shows that the $s p$ band can be traced through the $d$ resonances, although strongly damped. The limit for crossing thus occurs for lower values of $D$ in $A(k, E)$ than in $E_{\mathrm{r}}(k)$. This shows the importance of using $A(k, E)$ rather than $E_{\mathrm{r}}(k)$ when analysing experiments. Durham et al. [9] have shown that it is relevant to consider $A(k, E)$ also when analysing photoemission spectra, which is not a trivial fact.

We stress that the present model is only qualitative. We believe, however, that it accounts for some important qualitative concepts, which are of help at the interpretation of experimental results.

\section{Discussion}

Angle-resolved photoemission, as applied here, does not determine the full 3 -dimensional band structure. Although we only measure the parallel component of the $k$ vector, Fig. 2 provides the qualitative features of the bands.

The main interest in this work is to investigate the details in the interaction between the host band and the impurity $d$ band. Our model calculations indi- 
cate that the occurrence of band crossing between the $s p$ band and $t_{2 \mathrm{~g}} d$ states depends on the value of $d$ band damping $V_{d}$ relative to the hybridization term $2 W$. Detailed KKR-CPA calculations on $\mathrm{Ag}_{0.8} \mathrm{Pd}_{0.2}$ [7] and on $\mathrm{Cu}_{0.77} \mathrm{Ni}_{0.23}$ [8] show a clear crossing of the bands, but the $s$ band is strongly damped in this region. This situation corresponds roughly to $D$ larger than 0.3 in our model. Our experimental results show however a clear hybridization effect corresponding to $D=01 \div 0.2$. This fact points to the possibility that the representation of the random hybridization in the KKR-CPA calculations is not accurate enough. There are other photoemission experiments in literature on $\mathrm{Ag}_{x} \mathrm{Pd}_{1-x}[10,11]$ and similar alloys, but the $s p$ band dispersion has not been published.

The present problem of random hybridization should be of interest as test of details of the theory of disordered alloys, such as the description of broadening effects. Also, the degree of hybridization is of more general interest. It is an important ingredient for understanding of many phenomena, e.g. phase stability and RKKY interaction in spin-glass alloys.

\section{Conclusions}

The electronic structure of substitutionally disordered single crystals of $\mathrm{Ag}_{x} \mathrm{Pd}_{1-x}$ was studied by angle-resolved photoelectron spectroscopy. It was found that for concentrations $x \approx 90 \%$ the $\mathrm{Ag} 5 s p$ band hybridizes with the $\mathrm{Pd} 4 d t_{2 \mathrm{~g}}$ state. The $e_{\mathrm{g}}$ state is unaffected, due to symmetry reasons. CPA calculations in literature indicate however that the $s p$ band crosses the $d$ band. This raises the question if the present CPA calculations do not represent the random hybridization accurately enough. In parameterized model calculations we were able to reproduce the experimental findings qualitatively, by choosing a small enough damping of the Pd $d$ state.

\section{Acknowledgments}

We wish to thank J. Kanski and H. Starnberg for valuable discussions. The work is supported by the Swedish Natural Science Research Council.

\section{References}

[1] The Electronic Structure of Complex Systems, Eds. P. Phariseau, W.M. Temmerman, Plenum Press, New York 1984.

[2] P. Soven, Phys. Rev. 156, 809 (1967).

[3] B.L. Györffy, G.M. Stocks, in: Electrons in Disordered Metals and at Metallic Surfaces, Eds. P. Phariseau, B.L. Györffy, L. Scheire, Plenum Press, New York 1979, p. 89.

[4] G.M. Stocks, H. Winter, in: The Electronic Structure of Complex Systems, Eds. P. Phariseau, W.M. Temmerman, Plenum Press, New York 1984, p. 463.

[5] J. Friedel, Nuovo Cimento Suppl. 7, 287 (1958).

[6] C. Norris, P.O. Nilsson, Solid State Commun. 6, 649 (1968). 
[7] A.J. Pindor, W.M. Temmerman, B.L. Györffy, G.M. Stocks, J. Phys, F, Met. Phys. 10, 2617 (1980).

[8] N.K. Allen, P.J. Durham, B.L. Györffy, R.G. Jordan, J. Phys. F, Met. Phys. 13, 223 (1983).

[9] P.J. Durham, in: The Electronic Structure of Complex Systems, Eds. P. Phariseau, W.M. Temmerman, Plenum Press, New York 1984, p. 709.

[10] A.D. McLachlan, J.G. Jenkin, R.C.G. Leckey, J. Liesegang, J. Phys. F, Met. Phys. 5, 2415 (1975).

[11] M. Pessa, H. Asonen, M. Lindroos, A.J. Pindor, B.L. Györffy, W. Temmermann, J. Phys. F, Met. Phys. 11, L33 (1981). 